\title{
Discrete Spectral Local Measurement Method for Testing Solar Concentrators
}

\author{
Huifu Zhao, ${ }^{1,2}$ Hua Liu, ${ }^{1}$ Lei Jing, ${ }^{1,2}$ Ying Liu, ${ }^{1}$ Chun Li, ${ }^{1}$ Zhenwu Lu, ${ }^{1}$ and Qiang Sun ${ }^{1}$ \\ ${ }^{1}$ Opto-Electronics Technology Center, Changchun Institute of Optics, Fine Mechanics and Physics, Chinese Academy of Sciences, \\ Changchun 130033, China \\ ${ }^{2}$ Graduate School of the Chinese Academy of Sciences, Beijing 100039, China
}

Correspondence should be addressed to Hua Liu, liuhuarain@yahoo.com.cn

Received 28 July 2011; Accepted 29 September 2011

Academic Editor: Songyuan Dai

Copyright () 2012 Huifu Zhao et al. This is an open access article distributed under the Creative Commons Attribution License, which permits unrestricted use, distribution, and reproduction in any medium, provided the original work is properly cited.

\begin{abstract}
In order to compensate for the inconvenience and instability of outdoor photovoltaic concentration test system which are caused by the weather changes, we design an indoor concentration test system with a large caliber and a high parallelism, and then verify its feasibility and scientificity. Furthermore, we propose a new concentration test method: the discrete spectral local measurement method. A two-stage Fresnel concentration system is selected as the test object. The indoor and the outdoor concentration experiments are compared. The results show that the outdoor concentration efficiency of the two-stage Fresnel concentration system is $85.56 \%$, while the indoor is $85.45 \%$. The two experimental results are so close that we can verify the scientificity and feasibility of the indoor concentration test system. The light divergence angle of the indoor concentration test system is $0.267^{\circ}$ which also matches with sunlight divergence angle. The indoor concentration test system with large diameter (145 mm), simple structure, and low cost will have broad applications in solar concentration field.
\end{abstract}

\section{Introduction}

Solar energy is a kind of renewable, clean energy which is largest and most widely distributed in the world [1]. Its main applications are the thermal power generation $[2,3]$ and the photovoltaic power generation $[4,5]$. However, the high cost of photovoltaic power generation leads to its failure to dominate the market. The most effective way to reduce the cost of photovoltaic power generation is to use inexpensive optical concentration elements [6] to converge the sunlight to the small solar cells. This method can improve the concentration efficiency and reduce the cost of photovoltaic power generation. The Fresnel concentrator [7] with small size, light weight, and low cost can make optical concentration system to achieve miniaturization, integration, and economization. Therefore, the Fresnel concentrators have been widely used in solar energy fields.

The performance test of concentration system is crucial, because it can provide the feedback as the guide of design and manufacture. And we can improve the design and process according to the results. The currently test methods of concentration system are mainly the outdoor sunlight test [8] and the indoor solar simulator test. Although the outdoor test has real sunlight spectrum and light intensity distribution, the test results are unstable because of the influence of weather and environment. A solar simulator [9] is selected as the test source of indoor concentration test system. The solar simulator has been mainly used in solar cell test [10], space environment simulation, aging test, and so on. Because of the complexity, high cost, and large light divergence angles $\left(6^{\circ} \sim 10^{\circ}\right)$ of the solar simulator, it is not suitable for testing photovoltaic concentration systems. Up to now, there is no special equipment for testing the concentration system in the market. In this paper, we design an indoor concentration test system with large diameter $(145 \mathrm{~mm})$ and high parallelism (light divergence angle is $0.267^{\circ}$ ) which can test the performance of concentration system. Thus, the indoor concentration test system will have broad applications in the concentration photovoltaic field. 


\section{Indoor Concentration Test System and Discrete Spectral Local Measurement Method}

2.1. Design of Indoor Concentration Test System. The indoor concentration test system is composed of a light source, filters, a collimator, a stop, and a detector, as shown in Figure 1. The combination of a filter and the light source produces a monochromatic light, and we can adjust the collimator (see Figure $2, \tan \theta=b / a$ ) and make the divergence angle of exit light equal to $0.267^{\circ}\left(\theta=0.267^{\circ}\right)$. Concentration system converges the collimated rays to the detector, and we can obtain the power and illumination distribution of concentration spot from the detector.

2.2. Discrete Spectral Local Measurement Method. In this paper, we present a new concentration measurement method: the discrete spectral local measurement method. Most energy of the sun is concentrated in the spectral range from $350 \mathrm{~nm}$ to $1850 \mathrm{~nm}$, we choose wide-spectrum light source and narrow band filters to produce monochromatic light. The wavelength of monochromatic light distributes in the range from $350 \mathrm{~nm}$ to $1850 \mathrm{~nm}$. The weight of measured ring is the ratio of the area of measured ring to the whole area of Fresnel concentrator. The superposition of the product of the local concentration efficiency of monochromatic light and the weight of corresponding measured ring is defined as the average concentration efficiency of monochromatic light. The superposition of the average concentration efficiency multiplied by corresponding normalized weight of a variety of monochromatic light in the solar spectrum equals the whole concentration efficiency. We may measure the concentration efficiency of solar concentration system by using indoor concentration test system. We can analyze the scientificity and feasibility of the discrete spectral local measurement method through experimental test results compared with the theoretical simulation results.

\section{Software Simulation}

3.1. Theoretical Analysis of Concentration Ratio of Nonimaging Optics. The concentration ratio of nonimaging optics is divided into geometry concentration ratio $\left(C_{g}\right)$ and optical concentration ratio $\left(C_{o}\right)$. Geometry concentration ratio is defined as the ratio of the incident aperture area $\left(S_{1}\right)$ and the exit aperture area $\left(S_{2}\right)$ of the concentration system. Concentration efficiency $(\eta)$ is the ratio of the optical power $\left(\phi_{2}\right)$ at the exit aperture and the optical power $\left(\phi_{1}\right)$ at the incident aperture. Geometry concentration ratio $\left(C_{g}\right)$ multiplying concentration efficiency $(\eta)$ equals the optical concentration ratio $\left(C_{o}\right)[11]$, as shown in Figure 3

$$
\begin{gathered}
C_{g}=\frac{S_{1}}{S_{2}}, \\
\eta=\frac{\phi_{2}}{\phi_{1}}, \\
C_{o}=\frac{\phi_{2} / S_{2}}{\phi_{1} / S_{1}}=C_{g} * \eta .
\end{gathered}
$$

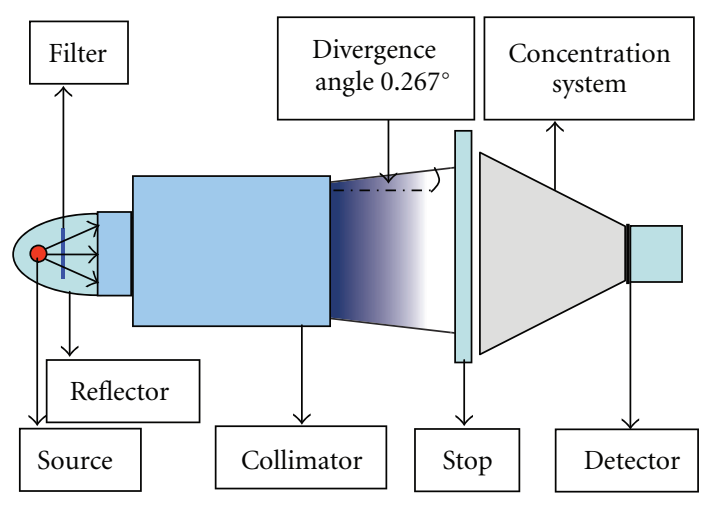

FIGURE 1: Schematic diagram of concentration test system.

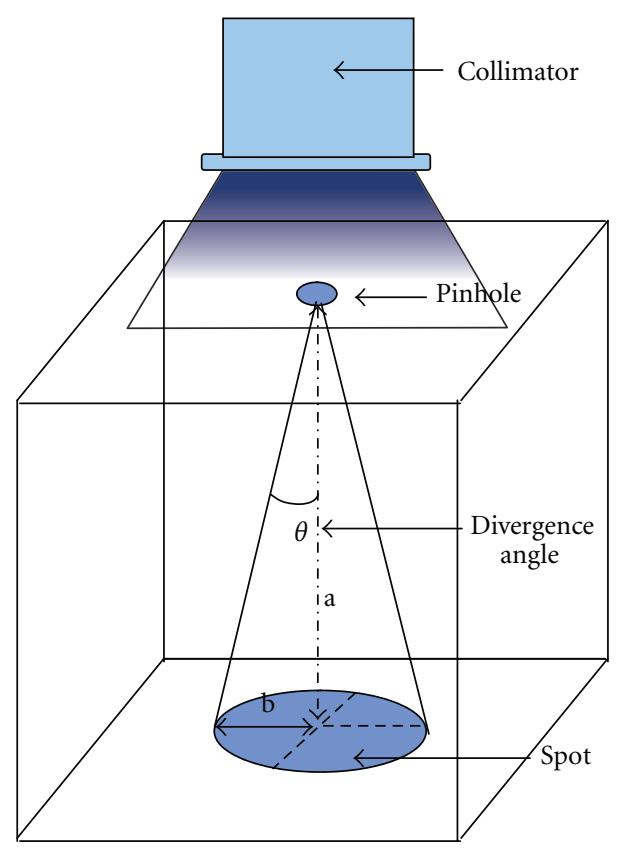

FIgURE 2: Schematic diagram of regulation collimator.

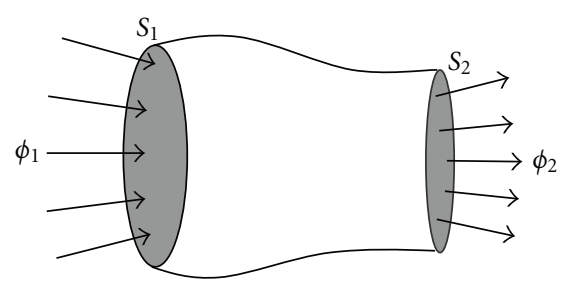

FIgURE 3: Schematic diagram of geometrical concentration ratio and optical concentration ratio.

The two-stage Fresnel concentration system which is composed of Fresnel concentrator and light funnel is selected as tested object. We model the two-stage Fresnel concentration system by the optical analysis software. The material of Fresnel concentrator is polymethylmethacrylate (PMMA) with a refractive index of 1.5, a relatively low density, high transmittance (The transmittance is higher than $92 \%$ in the scope 
of $300 \mathrm{~nm}$ to $2000 \mathrm{~nm}$ wavelength), excellent optical performance, and lower cost. The light funnel can collect all of the concentrated rays, so it will avoid energy loss. The geometrical concentration ratio of the two-stage Fresnel concentration system is $165 \mathrm{X}$ according to (1). The nonimaging Fresnel concentration system $[12,13]$ has no fixed focus, and it does not require image quality and rectification aberrations; however, it need to pay more attention to the concentration efficiency and the irradiation distributions of concentration spot.

3.2. The Distribution of Concentration Spot. We select LED as the source of the concentration test system. The spectral range of LED is $400 \mathrm{~nm} \sim 800 \mathrm{~nm}$. Monte Carlo ray tracing method is used for analysis concentration efficiency and irradiation distributions of concentration spot. The simulation results are shown in Figures 4 and 5.

The concentration efficiency of the two-stage Fresnel concentration system is $88.623 \%$. The optical concentration ratio of the two-stage Fresnel concentration system is $146 \mathrm{X}$ according to (3). $X$-axis of Figure 5 represents the position coordinates of vertical and horizontal directions which pass though the center of the receiver and the unit is millimeter. $Y$-axis represents the value of irradiation distributions; the unit is $\mathrm{W} / \mathrm{m}^{2}$. The blue curve represents irradiation distributions curve in the horizontal direction. The green curve represents irradiation distributions curve in the vertical direction. It can be seen from Figure 5 that the uniformity of central region $(-2 \mathrm{~mm} \sim 2 \mathrm{~mm})$ is $96 \%$ according to (4), where $U$ : the uniformity, $E_{\text {ave }}$ : average illumination, and $E_{\max }$ : $\max$ illumination, so it is very uniform. The irradiation value decreases from the center to the edge of the receiver.

$$
U=\frac{E_{\mathrm{ave}}}{E_{\max }} .
$$

3.3. The Transmittance and Concentration Efficiency of Several Monochromatic Lights. We choose a wide spectrum source and several narrow band filters to simulate the monochromatic light. LED with $400 \mathrm{~nm} \sim 800 \mathrm{~nm}$ spectral scope is selected as the source according to the laboratory existing conditions. Several monochromatic lights are obtained, respectively, as the source of concentration test system to simulate the concentration efficiency. Firstly, $423 \mathrm{~nm}$ monochromatic light is selected as a simulation source, and then, analysis of local concentration efficiency and transmittance of Fresnel concentration system by optical analysis software is done. The simulation results are shown in Table 1.

According to local transmittance, local concentration efficiency and their position weight, the average transmittance and concentration efficiency can be calculated as

$$
\begin{gathered}
T=\frac{\sum_{i=1}^{4} t_{i} * \pi\left(r_{i+1}^{2}-r_{i}^{2}\right)}{S}, \\
\eta=\frac{\sum_{i=1}^{4} \eta_{i} * \pi\left(r_{i+1}^{2}-r_{i}^{2}\right)}{S},
\end{gathered}
$$

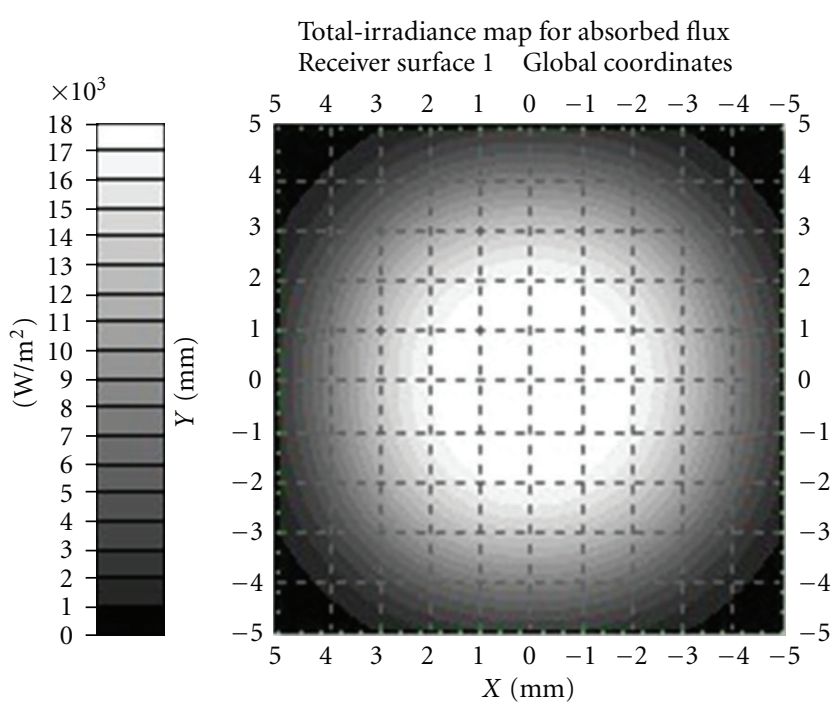

Irradiance min: $80.313 \mathrm{~W} / \mathrm{m}^{2}$, $\max : 17969 \mathrm{~W} / \mathrm{m}^{2}$, ave: $8862.3 \mathrm{~W} / \mathrm{m}^{2}$

RMS: 5808.7, normalized flux: 0.886231044091 incident rays

FIGURE 4: The illumination map on the receiver surface.

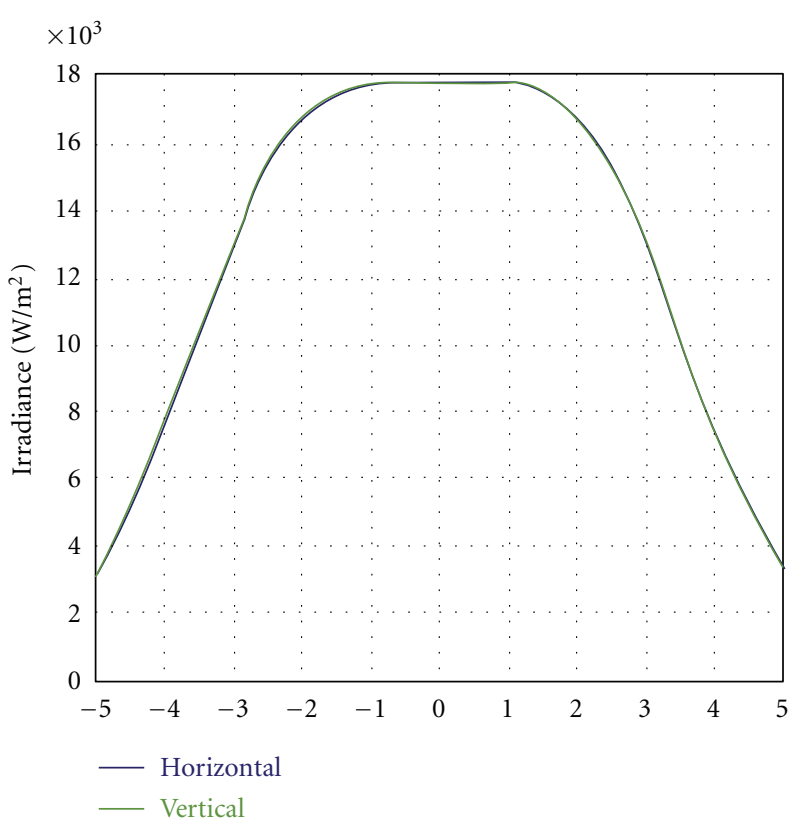

Horizontal/vertical axis of the receive (mm)

FIGURE 5: Illumination distribution curve on the receiver surface.

where $T$ is the average transmittance of monochromatic light in (5); $\eta$ is the average concentration efficiency of monochromatic light in $(6) ; t_{i}$ and $\eta_{i}$ are the average transmittance and concentration efficiency in the ring area from radius $r_{i}$ to $r_{i+1}$, respectively; $S$ represents the area of a circle of radius $70 \mathrm{~mm}$. $r_{1}=0 ; r_{2}=10 ; r_{3}=30 ; r_{4}=50 ; r_{5}=70$. The average transmittance is $91.10 \%$ according to (5). The average concentration efficiency is $89.57 \%$ according to (6). We use the same method to simulate the average transmittance and 
TABLE 1: The local transmittance and concentration efficiency of concentration system through software simulation.

\begin{tabular}{lcccccccccccccc}
\hline Position (mm) & $x=-60$ & $x=-40$ & $x=-20$ & $\begin{array}{c}\text { The } \\
\text { center }\end{array}$ & $x=20$ & $x=40$ & $x=60$ & $y=-60$ & $y=-40$ & $y=-20$ & $y=20$ & $y=40$ & $y=60$ & Average \\
\hline Transmittance \% & 91.02 & 91.15 & 91.22 & 91.27 & 91.22 & 91.14 & 91.02 & 91.03 & 91.13 & 91.25 & 91.25 & 91.15 & 91.02 & 91.10 \\
Efficiency \% & 89.24 & 90.77 & 88.31 & 88.58 & 88.30 & 90.77 & 89.24 & 89.23 & 90.78 & 88.30 & 88.29 & 90.76 & 89.24 & 89.57 \\
\hline
\end{tabular}

TABLE 2: The transmittance and concentration efficiency of concentration system for different monochromatic light.

\begin{tabular}{|c|c|c|c|c|c|c|c|}
\hline Wavelength (nm) & $423 \mathrm{~nm}$ & $490 \mathrm{~nm}$ & $510 \mathrm{~nm}$ & $525 \mathrm{~nm}$ & $615 \mathrm{~nm}$ & $700 \mathrm{~nm}$ & $785 \mathrm{~nm}$ \\
\hline Transmittance of simulation $\%$ & 91.10 & 91.21 & 91.21 & 91.21 & 91.22 & 91.22 & 91.22 \\
\hline Efficiency of simulation \% & 89.57 & 89.57 & 89.57 & 89.57 & 89.57 & 89.57 & 89.57 \\
\hline Normalized weight $W_{i}$ & 0.0649 & 0.1658 & 0.2325 & 0.3319 & 0.1817 & 0.0226 & 0.0006 \\
\hline
\end{tabular}

the concentration efficiency of other monochromatic light. The data is shown in the Table 2 as details.

\subsection{Verification of the Discrete Spectral Local Measurement} Method. The simulated concentration efficiency of Fresnel concentration system is $88.623 \%$ by the optical analysis software Tracepro, it is shown in Figure 4. The superposition of concentration efficiency of each monochromatic light multiplied by the weight $w_{i}$ equals the concentration efficiency of the whole spectral scope. The concentration efficiency is $89.57 \%$ according to (7)

$$
\eta=\sum_{i} \eta_{i} * w_{i}
$$

where, $\eta_{i}$ represents the average concentration efficiency of each monochromatic light; $w_{i}$ represents the weight of each monochromatic light in the whole spectrum. And their values are shown in Table 2.

The concentration efficiency of the two-stage Fresnel concentration system [14] by the discrete spectral local measurement method is higher than that by LED whole spectrum method according to the simulation results. The difference between them is $0.947 \%$. The deviation of them comes mainly from two aspects. Firstly, seven kinds of monochromatic light are selected only according to the existing conditions of laboratory. Small number of monochromatic light causes low accuracy. Secondly, sampling measured points are not enough (13 sampling measured points are selected), which lead to low measured accuracy also. If the number of monochromatic light and the sampling measured points are enough, the weight of monochromatic light and the average concentration efficiency will be more accurate.

\section{Experiments}

4.1. Indoor Concentration Test Process and Experimental Device. Figure 6 is the flow chart of the experimental test. LED source with $400 \mathrm{~nm} \sim 800 \mathrm{~nm}$ spectral scope and seven narrowband filters produce monochromatic light. We can control the divergence angle of incident light and make it to be $0.267^{\circ}$ by adjusting the collimator. The control process of divergence angle of incident light is shown in Figure 2. The focal length of collimator is that, $F=1600 \mathrm{~mm}, D / F=1 / 11$,

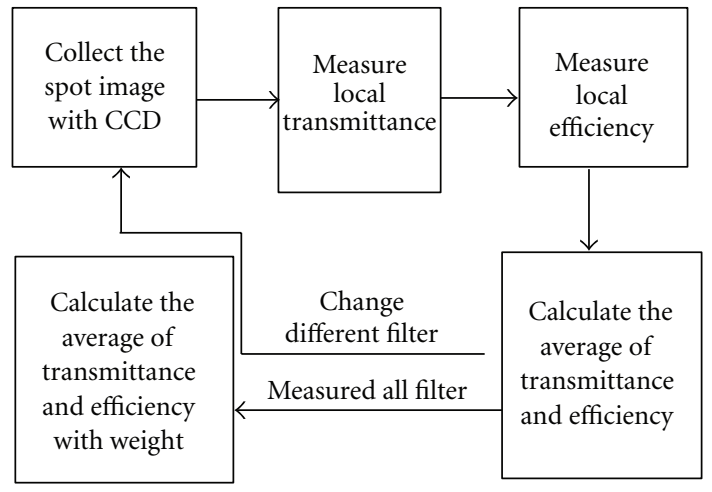

Figure 6: The flow chart of experimental test.

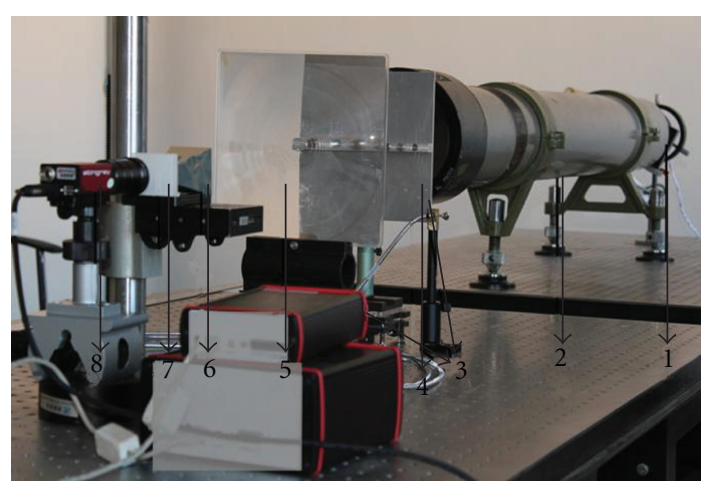

FIGURE 7: Physical map of indoor concentration test system. 1. LED source; filter, 2. collimator, 3. fiber spectrometer, 4. stop, 5. fresnel concentration system, 6. light funnel, 7. opal glass, and 8. CCD camera.

and the aperture $D=145 \mathrm{~mm}$. The indoor experimental devices are shown in Figure 7.

4.2. The Uniformity of Concentration Spot Distribution. The CCD camera (AVT Stingray F046B) is used to collect the image information of concentration spot. The strong irradiance of concentration spot makes CCD camera saturated so that it cannot accurately capture the image information. An opal glass (Edmund NT43-718) is placed at the focus plane which decreases the intensity of concentration spot to 
the same extent. The CCD camera is used to collect image information of concentration spot on the condition that the CCD camera does not reach saturation. The opal glass is a flat glass which is coated a layer opal film. There are some tiny particles in the opal film, and each tiny particle is equivalent to a point source when the tiny particle is illuminated. The light scattered from the particle which is lighted by the bright light is brighter than the other locations, and the light scattered by the particles superimposed on the receiver. So, the opal glass does not destroy holistic illumination distribution but makes local illumination uniformly distribution.

Figure 8 is the image of collected concentration spot; Figure 9 is a contour of concentration spot. The two axes denote the size of concentration spot in the Figure 9. Different colors represent different gray values. The irradiation distribution in the center of the concentration spot is relatively uniform, and the irradiation only decreases at the edge of the concentration spot.

The horizontal axis represents horizontal position coordinate of the concentration; the vertical axis represents the gray value of concentration spot in the Figure 10. The horizontal axis represents vertical position coordinate of the concentration; the vertical axis represents the gray value of concentration spot in the Figure 11. The illumination is relatively uniform in the central region $(-2 \mathrm{~mm} \sim 2 \mathrm{~mm})$ of concentration spot both in the horizontal and vertical direction. The Illumination decreases from the center region area with $\pm 2 \mathrm{~mm}$ to the edge of concentration spot. The experimental results are very close to the simulated results. The uniform distribution of central part avoids reducing the photoelectric conversion efficiency even damaging the solar cells which is caused by local parts of solar cell overheat.

\subsection{The Measurement of Transmittance and the Concentration} Efficiency by Indoor Concentration Test System. In order to measure local manufacture errors and production quality of concentration system, we need to test the local transmittance and the local concentration efficiency of the concentration system.

Step 1. The combination of LED source and $423 \mathrm{~nm}$ narrow band filter with full width at half maximum (FWHM) $5 \mathrm{~nm}$ produces monochromatic light.

Step 2. A stop with a row of holes in the horizontal direction and the vertical direction is set at $10 \mathrm{~mm}$ behind the exit aperture of collimator. The diameter of the hole is $4 \mathrm{~mm}$. The distance between adjacent holes is $20 \mathrm{~mm}$. The smaller are the diameter and the distance of holes, the more accurate are the local measurement results. Each time there is only one hole keeping open. So, it can reduce measurement errors caused by the position deviations.

Step 3. The Fresnel concentration system is set at $15 \mathrm{~mm}$ behind the spot. The detector is set at the focal point to test the concentration spot energy. The funnel is close to the detector to avoid energy leaking. The optical powermeter (Ophir PD300-1W) is used as the system detector, and the size of probe is $10 \mathrm{~mm} * 10 \mathrm{~mm}$. The optical powermeter

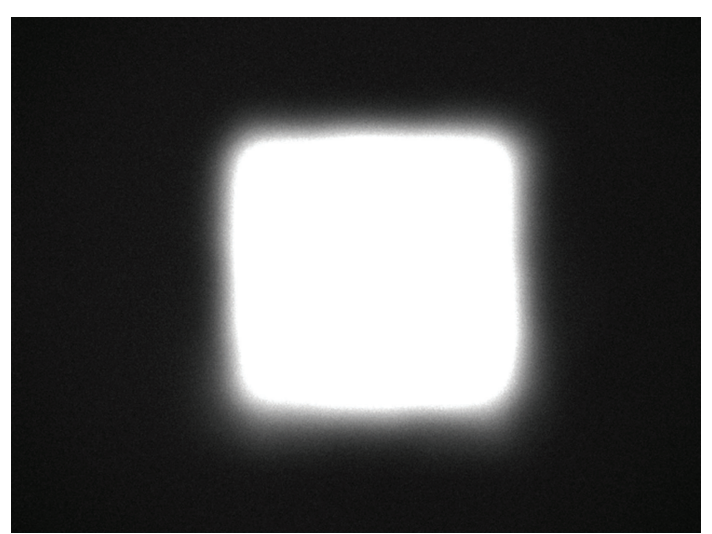

FIgURE 8: Concentration spot on the receiver.

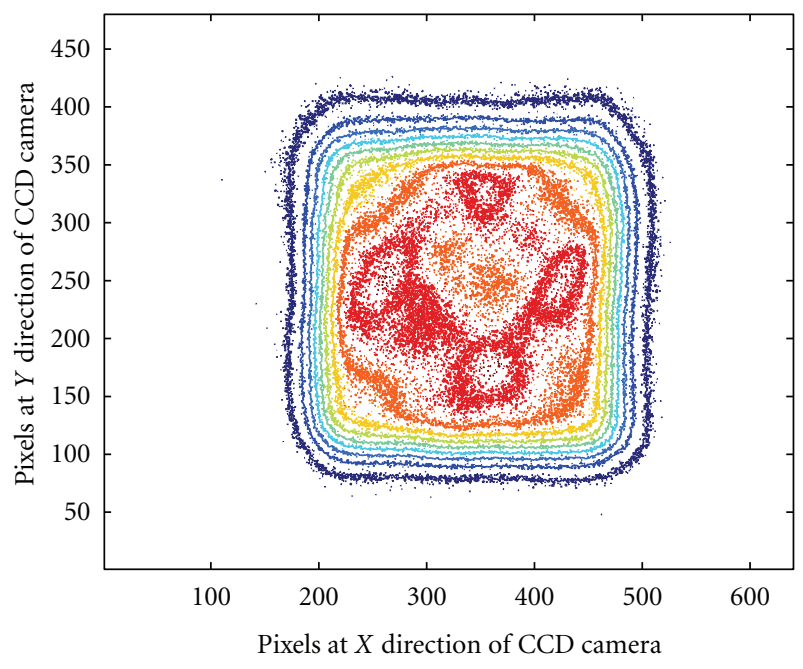

FIGURE 9: The contour of concentration spot.

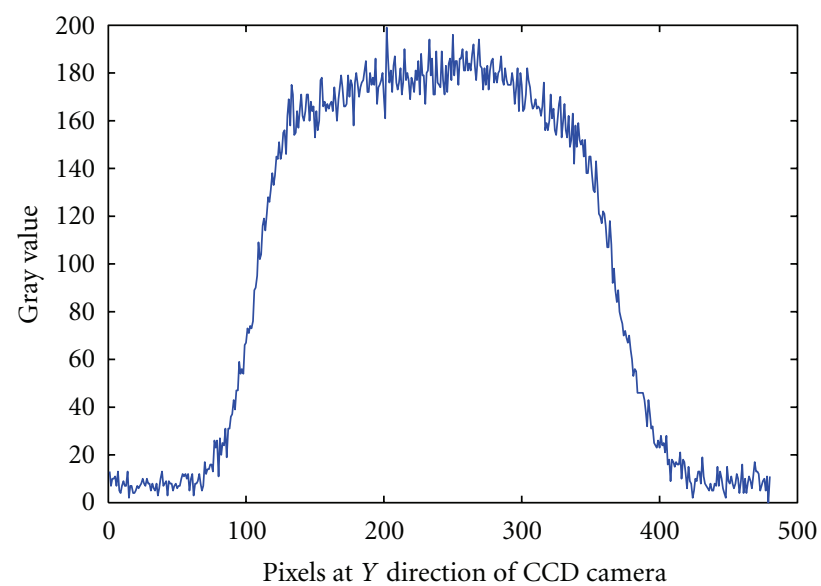

Figure 10: Horizontal illumination distribution of concentration spot on the receiver. 


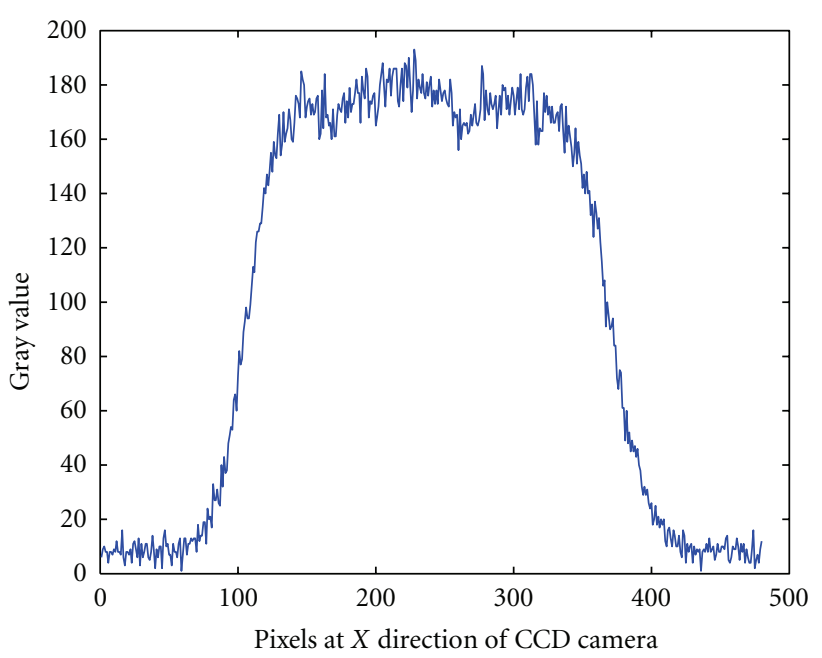

FIgURE 11: Vertical illumination distribution of concentration spot on the receiver.

is used to measure the optical power $\left(E_{i}\right)$ through each hole.

Step 4. Keep the source and the concentration system stationary and remove the optical powermeter to $10 \mathrm{~mm}$ behind the Fresnel contractor. The energy $\left(E_{o}\right)$ through each hole of the Fresnel concentrator and the energy $\left(E_{F}\right)$ concentrated by Fresnel concentrator are measured.

The transmittance of Fresnel concentration system is

$$
T=\frac{E_{o}}{E_{i}} .
$$

The concentration efficiency of Fresnel concentration system is

$$
\eta=\frac{E_{F}}{E_{i}} .
$$

The experiment measured average transmittance of Fresnel concentration for $423 \mathrm{~nm}$ wavelength is much close to the results of software simulation. The difference of them is $2.59 \%$. The difference of the concentration efficiency is about $3.94 \%$. The transmittance and the concentration efficiency in the ring region $20 \mathrm{~mm}$ away from the center of concentrator are obviously lower than other region. The detailed results are shown in Table 3. But the simulation results by software are almost same in all parts of Fresnel concentrator. So, we can conclude that the manufacture errors in the ring region $20 \mathrm{~mm}$ away from the center of concentrator are larger than any other region which leads to reduce the concentration efficiency of Fresnel concentration system. Using the same measurement method analysis, the concentration efficiency of other monochromatic light, the detailed results are shown in Table 4.

The experimental concentration efficiency is $85.45 \%$ according to (7). The optical concentration ratio of the two

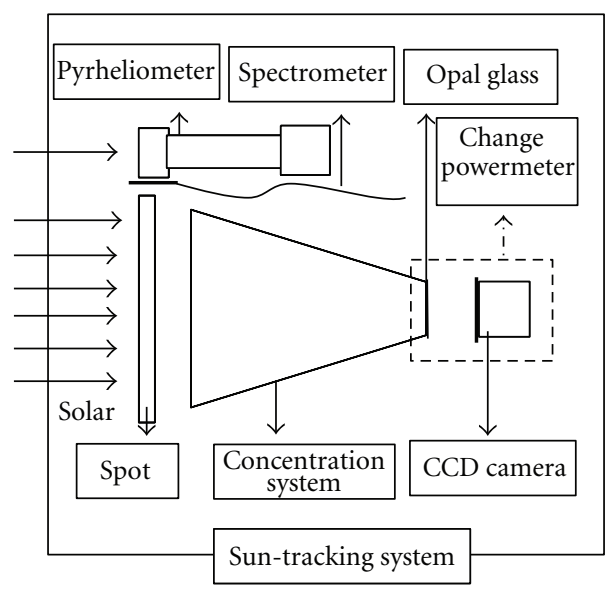

FIGURE 12: Schematic diagram of outdoor concentration test system.

stages Fresnel concentration system is $C_{o}=C_{g} * \eta=$ $165 * 85.45 \%=141$ according to $(3)$. The simulated concentration efficiency is $88.623 \%$ by optical analysis software. The difference between the simulation results and the experimental results is $3.173 \%$, and the difference is mainly caused by manufacture errors and measurement errors. In order to improve the efficiency of the concentration system and photoelectric conversion efficiency of solar cells, we will improve the design and manufacture process of the concentration system according to experimental test results.

4.4. The Measurement of Transmittance and the Concentration Efficiency by Outdoor Concentration Test System. We will further verify the scientificity of the discrete spectrum local measurement method and the indoor concentration test system through outdoor concentration test experiment. The outdoor concentration test system [8] is composed of a suntracking system, two-stage Fresnel concentration system (the same as indoor concentration test system), a pyrheliometer (Kipp and Zonen, model CHP1), a fiber spectrometer, a stop, a powermeter, a detector (CCD camera), a counter, and other devices, as shown in Figure 12.

An outdoor solar concentration test system is shown in Figure 13. The same two stages Fresnel concentration system is selected as the test object. The Fresnel concentrator converges the incident sunlight through the stop into the funnel. All rays are total internal reflected several times in the funnel, and finally, they are received by the detector. The funnel can converge the converged rays and make the collected rays distribute uniformly. We use the powermeter to measure the power of the converged rays. The power of incident rays is directly detected by the pyrheliometer. The ratio of them is the concentration efficiency of outdoor concentration system. The average concentration efficiency of the outdoor concentration test system is $85.56 \%$. The difference between the test results of outdoor test system and the of indoor's is $0.11 \%$ which is mainly caused by that the quantity of narrow band filter is not enough in the discrete spectral local measurement method. If the narrow band 
TABLE 3: The measured transmittance and the concentration efficiency of the concentration system.

\begin{tabular}{lcccccccccccccc}
\hline Position (mm) & $x=-60$ & $x=-40$ & $x=-20$ & $\begin{array}{c}\text { The } \\
\text { center }\end{array}$ & $x=20$ & $x=40$ & $x=60$ & $y=-60$ & $y=-40$ & $y=-20$ & $y=20$ & $y=40$ & $y=60$ & Average \\
\hline Transmittance \% & 88.46 & 90.16 & 86.36 & 92.50 & 87.88 & 89.68 & 86.72 & 89.84 & 89.26 & 85.25 & 86.05 & 90.52 & 87.50 & 88.51 \\
Efficiency \% & 87.31 & 87.25 & 82.48 & 87.00 & 82.24 & 87.71 & 86.38 & 82.03 & 87.12 & 82.59 & 82.87 & 87.21 & 86.17 & 85.63 \\
\hline
\end{tabular}

TABLE 4: Experimental transmittance and concentration efficiency of concentration system for different monochromatic light.

\begin{tabular}{|c|c|c|c|c|c|c|c|}
\hline Wavelength (nm) & $423 \mathrm{~nm}$ & $490 \mathrm{~nm}$ & $510 \mathrm{~nm}$ & $525 \mathrm{~nm}$ & $615 \mathrm{~nm}$ & $700 \mathrm{~nm}$ & $785 \mathrm{~nm}$ \\
\hline Transmittance of experiment \% & 88.51 & 88.58 & 88.57 & 88.60 & 88.59 & 88.58 & 88.57 \\
\hline Efficiency of experiment \% & 85.63 & 85.51 & 85.50 & 85.51 & 85.19 & 85.08 & 84.96 \\
\hline Normalized weight $W_{i}$ & 0.0649 & 0.1658 & 0.2325 & 0.3319 & 0.1817 & 0.0226 & 0.0006 \\
\hline
\end{tabular}

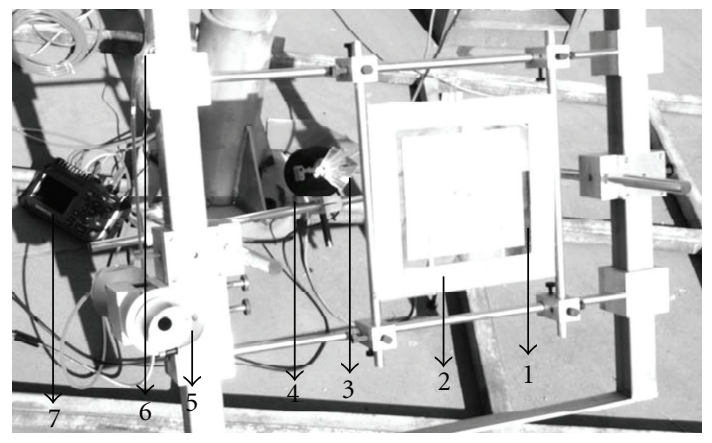

FIGURE 13: Physical map of concentration test system. 1. Fresnel concentrator, 2. stop, 3. light funnel, 4. powermeter, 5. pyrheliometer, 6. fiber spectrometer, and 7. arithmometer.

filters are enough and uniformly distribute in the scope of sun spectrum, the concentration efficiency by the two kinds test method will be very close. Therefore, the discrete spectral local measurement method and indoor concentration test system are both scientific and feasible.

\section{Conclusions}

We design the indoor concentration test system and propose a new measurement method: the discrete spectral local measurement method. We verify the scientificity of the indoor test system and the discrete spectral local measurement method through comparative analysis of the simulation and experimental results. We employ the wide-spectrum source and enough filters to simulate the sunlight which is then used as the source of the indoor concentration test system. The discrete spectral local measurement method can test local manufacture quality of Fresnel concentrator. We can modify the design and manufacture process to improve the concentration efficiency according to test results. The indoor concentration test system avoids the measurement instability which is caused by changing weather in the outdoor concentration test system. The indoor concentration test system can accurately evaluate the performance of the concentration system. Therefore, the indoor concentration test system will promote the further development of PV industry.

\section{Acknowledgments}

The authors gratefully acknowledge the financial support from National Basic Research Program of China with Grant no. 2010CB227101 and from the Innovation Program of Chinese Academy of Sciences. Supported by Natural Science Foundation of Jilin under Grant no. 201115123.

\section{References}

[1] B. S. William and G. Micheal, "Power from the sun[M/OL]," 2001, http://www.powerfromthesun.net/book.html.

[2] X. Wei, Z. Lu, Z. Lin, and Z. Wang, "Design and optimization of heliostat field layout for solar tower power plant," Acta Optica Sinica, vol. 30, no. 9, pp. 2652-2656, 2010.

[3] R. T. Wang and X. D. Wei, "Shadow of heliostat fleld in the solar tower power plant," Acta Photonica Sinica, vol. 38, no. 9, pp. 2414-2418, 2009.

[4] R. Winston, J. C. Miñano, and P. G. Benítez, Nonimaging Optics, Elsevier Academic Press, 2004.

[5] D. Liu, H. Qiao, L. Kong, and J. Jia, "The study on R-V chacteristics of HgCdTe photovoltaic detectors under different baked temperature," Acta Optica Sinica, vol. 29, pp. 336-339, 2009.

[6] W. T. Welford and R. Winston, High Collection Non-Imaging Optics, Academic Press, New York, NY, USA, 1989.

[7] M. D. Watson and R. R. Jayroe, "Fresnel lens solar concentrator design based on geometric optics and blackbody radiation equation," in Proceedings of the SPIE-Nonimaging Optics: Maximum Efficiency Light Transfer V, vol. 3781, pp. 85-93, July 1999.

[8] D. Chemisana, A. Vossier, A. Dollet, B. A. Mesih, J. Rosell, and M. Ibáñez, "A characterization method for testing solar concentrators using a lambertian diffuser," http://www.concentrating-pv.org/darmstadt2009/pdf/papers/14-ChemisanaEtAlLambertianTestForConcentrator.pdf.

[9] H. Pang, B. Huang, Y. Zang, and J. Chen, "Design of KM6 solar simulator," Spacecraft Environment Engineering, vol. 23, no. 3, pp. 125-133, 2006.

[10] Z. M. Wang, Z. B. Gong, G. P. Wei, and L. M. Liu, "Solar simulation technique for solar cell measurement," Optics and Precision Engineering, vol. 17, no. 7, pp. 1542-1547, 2009.

[11] J. S. Lin, W. C. Huang, H. C. Hsu, M. W. Chang, and C. P. Liu, "A study for the special Fresnel lens for high efficiency solar concenrators," in Proceedings of the SPIE-The International Society for Optical Engineering, vol. 5942, pp. 59420X-059420X-9, 2005. 
[12] H. Liu and Z. W. Lu, "Lateral splittable non-imaging concentrators with large acceptable angles," Optics and Precision Engineering, vol. 17, no. 12, pp. 2881-2886, 2009.

[13] R. Zhu, Z. W. Lu, H. Liu, and H. X. Zhang, "The solar concentrator design method based on nonimaging optics," Acta Photonica Sinica, vol. 31, no. 8, pp. 45-47, 2008.

[14] Y. M. Han, R. Z. Wang, Y. J. Dai, and A. H. Xiong, "Studies on the light permeance characteristic of a Fresnel lens group applied in high concentration solar energy," Journal of Optics A, vol. 9, no. 11, pp. 988-997, 2007. 


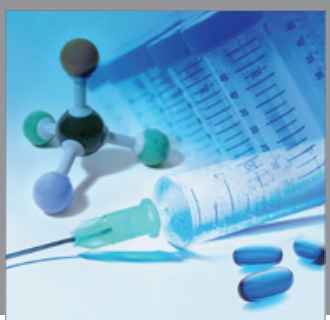

International Journal of

Medicinal Chemistry

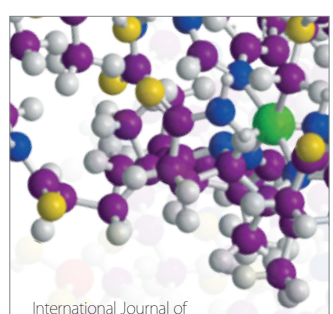

Carbohydrate Chemistry

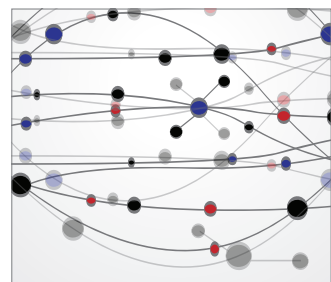

The Scientific World Journal
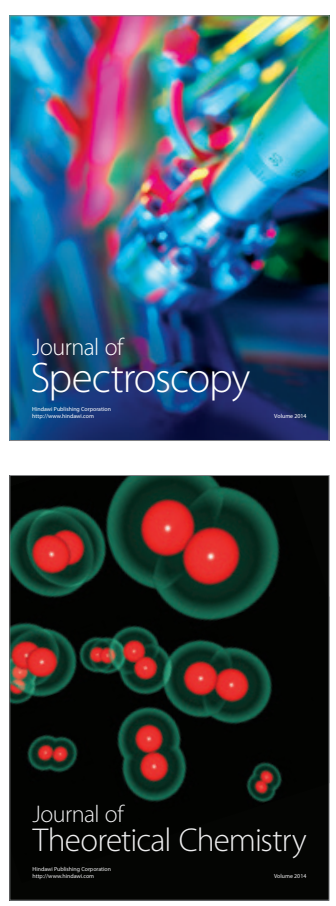
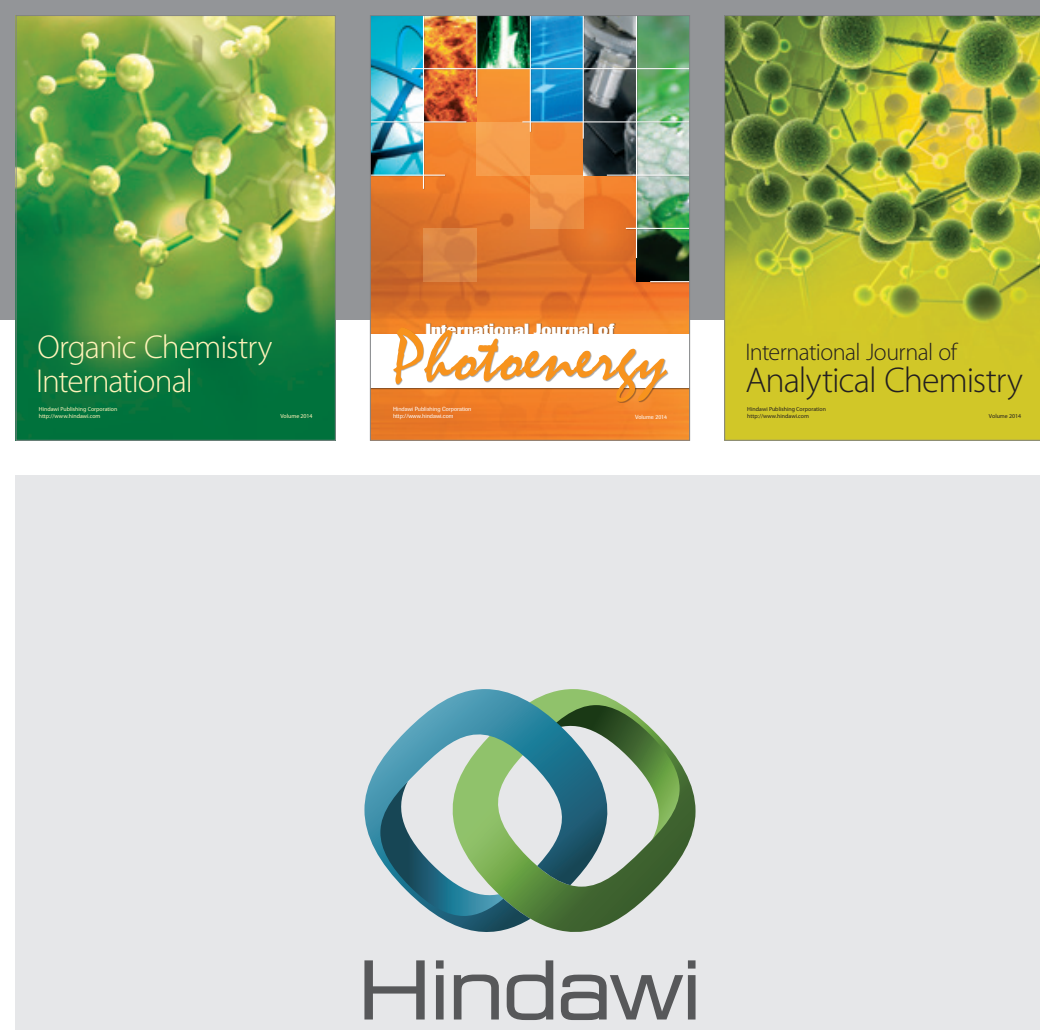

Submit your manuscripts at

http://www.hindawi.com
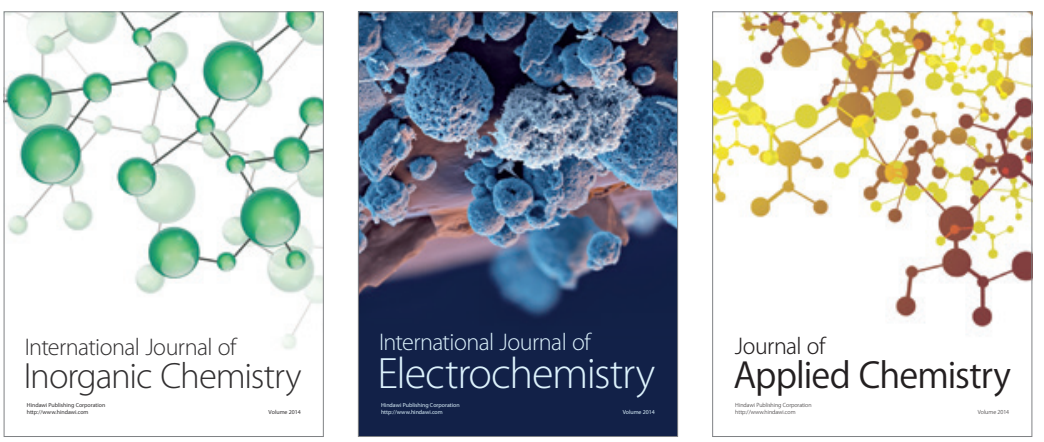

Journal of

Applied Chemistry
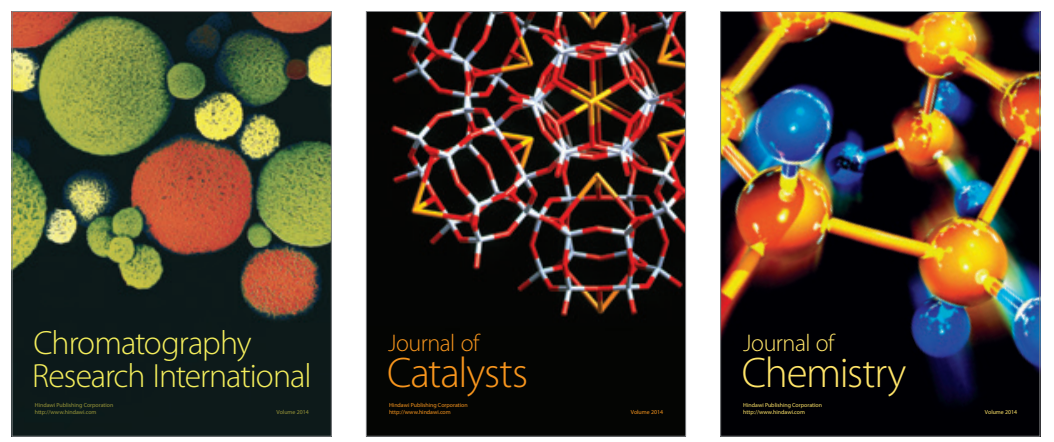
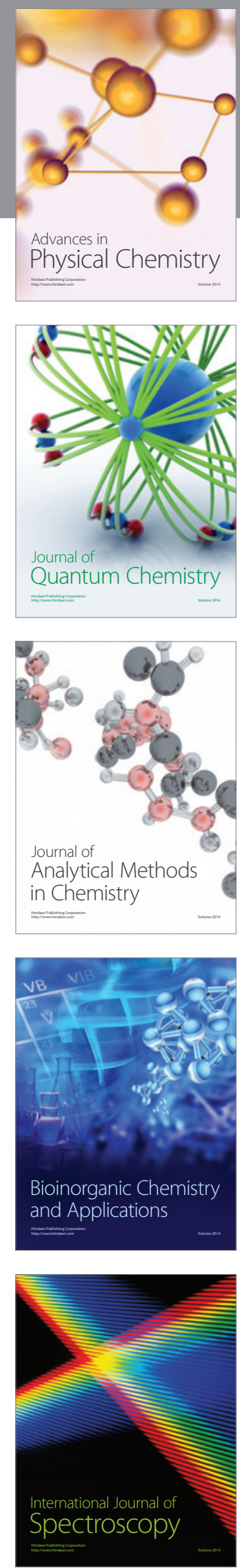